Pacific Journal of Mathematic 


\section{SPECTRAL INEQUALITIES INVOLVING THE INFIMA AND SUPREMA OF FUNCTIONS}

\section{Kong-Ming Chong}

This paper presents some spectral inequalities from which some useful tools for the investigations of the permanents of nonnegative matrices are derived as particular cases.

Introduction. In this paper, we establish some spectral inequalities involving the infima and suprema of functions as well as their equimeasurable rearrangements, and obtain as particular cases some rearrangement inequalities which turn out to be useful tools for the investigations of the permanents of non-negative matrices (see [4] and [5]).

1. Preliminaries. Let $M(X, \mu)$ denote the set of all extended real valued measurable functions defined on a finite measure space $(X, \Lambda, \mu)$. Two functions $f \in M(X, \mu)$ and $g \in M\left(X^{\prime}, \mu^{\prime}\right)$, where $\mu^{\prime}\left(X^{\prime}\right)=\mu(X)$, are said to be equimeasurable (written $f \sim g$ ) whenever

$$
\mu(\{x: f(x)>t\})=\mu^{\prime}(\{x: g(x)>t\})
$$

for all real $t$. If $f \sim g$, it follows from (1.1) that

$$
\Phi(f) \sim \Phi(g)
$$

whenever $\Phi: R \rightarrow R$ is a Borel measurable function.

If $f \in M(X, \mu)$, let $\delta_{f}:[0, \mu(X)] \rightarrow \bar{R}$ denote the decreasing rearrangement of $f$. It is clear that the function $\iota_{f}=-\delta_{-f}$, called the increasing rearrangement of $f$, satisfies $\iota_{f} \sim f$.

It is a direct consequence of (1.2) that

$$
\delta_{\psi(f)}=\psi\left(\delta_{f}\right) \quad\left(\text { respectively } \iota_{\psi(f)}=\psi\left(\iota_{f}\right)\right)
$$

whenever $\psi: R \rightarrow R$ is a left continuous (respectively right continuous) and non-decreasing function. Moreover, it is not hard to see that the operation of decreasing or increasing rearrangements preserves a.e. pointwise convergence, convergence in measure and all $L^{p}$ convergence, $1 \leqq p \leqq \infty$.

If $f, g \in M(X, \mu) \cup M\left(X^{\prime}, \mu^{\prime}\right)$ and $f^{+}, g^{+} \in L^{1}(X, \mu) \cup L^{1}\left(X^{\prime}, \mu^{\prime}\right)$, then we write $f \ll g$ whenever

$$
\int(f-t)^{+} \leqq \int(g-t)^{+}
$$

for all $t \in R$ and $f \prec g$ whenever $f \ll g$ and $\int f=\int g$. 
In the sequel, expressions of the form $f \prec g$ (respectively $f \ll g$ ) are called strong (respectively weak) spectral inequalities.

2. Some spectral inequalities. In what follows, for any given $n$-tuple $\boldsymbol{x}=\left(x_{1}, x_{2}, \cdots, x_{n}\right) \in R^{n}$, the $n$-tuples $\boldsymbol{x}^{*}=\left(x_{1}^{*}, x_{2}^{*}, \cdots, x_{n}^{*}\right)$ and $\boldsymbol{x}^{\prime}=\left(x_{1}^{\prime}, x_{2}^{\prime}, \cdots, x_{n}^{\prime}\right)$ respectively denote the decreasing and increasing rearrangements of $\boldsymbol{x}$.

Lemma 2.1. If $\left(a_{1}, a_{2}, \cdots, a_{n}\right) \in R^{n}$ and $\left(b_{1}, b_{2}, \cdots, b_{n}\right) \in R^{n}$, then

$$
\sum_{i=1}^{n}\left(a_{i}^{*} \wedge b_{i}^{\prime}\right)^{+} \leqq \sum_{i=1}^{n}\left(a_{i} \wedge b_{i}\right)^{+} \text {. }
$$

Proof. Since $\left(\boldsymbol{a}^{*}\right)^{+}=\left(\boldsymbol{a}^{+}\right)^{*},\left(\boldsymbol{b}^{\prime}\right)^{+}=\left(\boldsymbol{b}^{+}\right)^{\prime}$, by (1.3), and since $(r \wedge s)^{+}=r^{+} \wedge s^{+}$for all $r, s \in R$, we need only prove the lemma for $a_{i} \geqq 0$ and $b_{i} \geqq 0, i=1,2, \cdots, n$.

Without loss of generality, we may assume that $b_{i}=b_{i}^{\prime}, i=1$, $2, \cdots, n$. In this case, if $1 \leqq i<j \leqq n$ and $a_{i}<a_{j}$, then it is easily seen that $a_{i} \wedge b_{j}+a_{j} \wedge b_{i} \leqq a_{i} \wedge b_{i}+a_{j} \wedge b_{j}$. Thus, for each pair of integers $i, j$ such that $1 \leqq i<j \leqq n$, if $a_{i}<a_{j}$, the right-hand sum of the asserted inequality is never increased on interchanging $a_{i}$ and $a_{j}$. We, therefore, conclude that the left-hand sum of (2.1) is the smallest possible value attainable by the right-hand sum as $a$ ranges through all its rearrangements.

In the sequel, we denote the Lebesque measure on $R$ by $m$.

Lemma 2.2. If $f, g \in L^{1}(X, \Lambda, \mu)$ where $\mu(X)=a<\infty$, then

$$
\int_{0}^{a}\left(\delta_{f} \wedge \iota_{g}\right)^{+} d m \leqq \int_{X}(f \wedge g)^{+} d \mu .
$$

Proof. If the measure space $(X, \Lambda, \mu)$ is non-atomic, then there exist sequences $\left\{f_{n}\right\}_{n=1}^{\infty},\left\{g_{n}\right\}_{n=1}^{\infty}$ of simple functions with the same sets of constancy such that $f_{n} \rightarrow f$ and $g_{n} \rightarrow g$ both pointwise $\mu$-a.e. and in $L^{1}$. Since decreasing and increasing rearrangements preserve a.e. pointwise convergence and $L^{p}$ convergence, $1 \leqq p \leqq \infty$, we see that $\delta_{f_{n}}$ and $\iota_{g_{n}}$ also converge in $L^{1}$ to $\delta_{f}$ and $\iota_{g}$ respectively.

Now by Lemma 2.1, we have

$$
\int_{0}^{a}\left(\delta_{f_{n}} \wedge \iota_{g_{n}}\right)^{+} d m \leqq \int_{X}\left(f_{n} \wedge g_{n}\right)^{+} d \mu
$$

whence (2.2) follows by taking limits and on observing that $f_{n} \wedge g_{n}=$ $f_{n}-\left(f_{n}-g_{n}\right)^{+}$.

If $(X, A, \mu)$ is not non-atomic, we can imbed it into a non-atomic 
measure space $(\bar{X}, \bar{\Lambda}, \bar{\mu})$ (For details of this device, we refer to [2, pp. 52-54]). Then, $f, g \in L^{1}(X, \mu)$ can be identified with $\bar{f}, \bar{g} \in L^{1}(X, \mu)$ by a map $h \rightarrow \bar{h}$ which is linear and satisfying $h \sim \bar{h}$ and $\overline{\Phi(h)}=\Phi(\bar{h})$ for every Borel measurable $\Phi: R \rightarrow R$. Thus

$$
f \wedge g \sim \overline{f \wedge g}=\overline{f-(f-g)^{+}}=\bar{f}-(\bar{f}-\bar{g})^{+}=\bar{f} \wedge \bar{g}
$$

and so

$$
\int_{0}^{a}\left(\delta_{f} \wedge \iota_{g}\right)^{+} d m=\int_{0}^{a}\left(\delta_{\bar{f}} \wedge \iota_{\bar{g}}\right)^{+} d m \leqq \int_{\bar{X}}(\bar{f} \wedge \bar{g})^{+} d \bar{\mu}=\int_{X}(f \wedge g)^{+} d \mu .
$$

THEOREM 2.3. If $f, g \in L^{1}(X, \mu)$, where $\mu(X)=a<\infty$, then

$$
\delta_{f} \wedge \iota_{g} \ll f \wedge g \ll \delta_{f} \wedge \delta_{g}
$$

where the strong spectral inequality $f \wedge g \prec \delta_{f} \wedge \delta_{g}$ (respectively $\delta_{f} \wedge \iota_{g} \prec f \wedge g$ ) holds if and only if $f \wedge g \sim \delta_{f} \wedge \delta_{g}$ (respectively $\left.\delta_{f} \wedge \iota_{g} \sim f \wedge g\right)$.

Let $\Phi: R \rightarrow R$ be any increasing and continuous function, then $\Phi\left(\delta_{f} \wedge c_{g}\right) \ll \Phi(f \wedge g) \ll \Phi\left(\delta_{f} \wedge \delta_{g}\right)$. If $\Phi$ is strictly increasing and convex such that $\Phi\left(\delta_{f} \wedge \delta_{g}\right) \in L^{1}([0, a], m)$ (respectively $\Phi(f \wedge g) \in L^{1}(X, \mu)$ ), then $\Phi(f \wedge g) \prec \Phi\left(\delta_{f} \wedge \delta_{g}\right)$ (respectively $\left.\Phi\left(\delta_{f} \wedge \iota_{g}\right) \prec \Phi(f \wedge g)\right)$ if and only if $f \wedge g \sim \delta_{f} \wedge \delta_{g}$ (respectively $\delta_{f} \wedge \iota_{g} \sim f \wedge g$ ).

Proof. The spectral inequality $\delta_{f} \wedge \iota_{g} \ll f \wedge g$ follows immediately from the preceding lemma since, on substituting $f-t, g-t$ (where $t \in R$ ) for $f, g$ respectively in (2.2), we have

$$
\begin{aligned}
\int_{0}^{a}\left(\delta_{f} \wedge \iota_{g}-t\right)^{+} d m & =\int_{0}^{a}\left(\delta_{f-t} \wedge \iota_{g-t}\right)^{+} d m \leqq \int_{X}[(f-t) \wedge(g-t)]^{+} d \mu \\
& =\int_{X}(f \wedge g-t)^{+} d \mu .
\end{aligned}
$$

The spectral inequality $f \wedge g \ll \delta_{f} \wedge \delta_{g}$ is obvious since $\delta_{f \wedge g} \leqq$ $\delta_{f} \wedge \delta_{g}$ by the monotonicity of the decreasing rearrangement operator $\delta$. Thus, if $f \wedge g \prec \delta_{f} \wedge \delta_{g}$, then $\int_{0}^{a} \delta_{f \wedge g} d m=\int_{0}^{a} \delta_{f} \wedge \delta_{g} d m$ which is the case if and only if $\delta_{f \wedge g}=\delta_{f} \wedge \delta_{g} m$-a.e. or, equivalently, $f \wedge g \sim$ $\delta_{f} \wedge \delta_{g}$.

Suppose the strong spectral inequality $\delta_{f} \wedge \iota_{g} \prec f \wedge g$ holds. Let $\Psi: R \rightarrow R$ be any strictly concave and increasing function such that $\Psi(x)=\log x$ for $x$ large enough and that $\Psi(x)$ approaches $x$ asymptotically as $x \rightarrow-\infty$ (the existence of such a function $\Psi$ is geometrically clear; if both $f$ and $g$ are non-negative, we simply choose $\Psi(x)=$ $\log (1+x), x \geqq 0)$. Then $\Psi(h)$ is integrable whenever $h \in L^{1}(X, \mu) \cup$ $L^{1}([0, a], m)$ since $\Psi^{+}(h)=\log h^{+} \leqq h^{+}$for $h^{+}$large enough and 
$h^{-}-\varepsilon \leqq \Psi^{-}(h) \leqq h^{-}+\varepsilon$ for $h^{-}$large enough and for some $\varepsilon>0$. By (1.3), we clearly have $\Psi\left(\delta_{f} \wedge \iota_{g}\right)=\Psi\left(\delta_{f}\right) \wedge \Psi\left(\iota_{g}\right)=\delta_{\psi(f)} \wedge \iota_{\psi(g)} \ll$ $\psi(f) \wedge \psi(g)=\psi(f \wedge g)$. Now it is easily seen that the inverse $\Phi$ of $\psi$ is strictly convex and increasing, and so, by (1.2) and [1, Theorem 2.3], we have $\Phi\left(\Psi\left(\delta_{f} \wedge \iota_{g}\right)\right)=\delta_{f} \wedge \iota_{g} \prec f \wedge g=\Phi(\Psi(f \wedge g)$ if and only if $\delta_{f} \wedge \iota_{g} \sim f \wedge g$.

The rest is easy by virtue of the above result, (1.3) and [1, Theorems 2.3, 2.5 and 2.8].

Corollary 2.4. If $f, g \in L^{1}(X, \mu)$ where $\mu(X)=a<\infty$, then

$$
-\left(\delta_{f} \vee \iota_{g}\right) \ll-(f \vee g) \ll-\left(\delta_{f} \vee \delta_{g}\right)
$$

where the strong spectral inequality $-\left(\delta_{f} \vee \iota_{g}\right) \prec-(f \vee g)$ (respectively $\left.-(f \vee g) \prec-\left(\delta_{f} \vee \delta_{g}\right)\right)$ holds if and only if $\delta_{f} \vee \iota_{g} \sim f \vee g$ (respectively $f \vee g \sim \delta_{f} \vee \delta_{g}$ ).

Let $\Phi: R \rightarrow R$ be any nonincreasing and continuous function, then

$$
\Phi\left(\delta_{f} \vee \iota_{g}\right) \prec \Phi(f \vee g) \prec \Phi\left(\delta_{f} \vee \delta_{g}\right) .
$$

If $\Phi$ is strictly decreasing and convex such that $\Phi\left(\delta_{f} \vee \delta_{g}\right) \epsilon$ $L^{1}([0, a], m)$ (respectively $\left.\Phi(f \vee g) \in L^{1}(X, \mu)\right)$, then $\Phi(f \vee g) \prec\left(\delta_{f} \vee \delta_{g}\right)$ (respectively $\Phi\left(\delta_{f} \vee \iota_{g}\right) \prec \Phi(f \vee g)$ ) if and only if $f \vee g \sim \delta_{f} \vee \delta_{g}$ (respectively $\delta_{f} \vee \iota_{g} \sim f \vee g$ ).

Proof. The result follows immediately from Theorem 2.3 on substituting $-f$ for $f$ and $-g$ for $g$.

It is now easy to derive the rearrangement inequalities of Jurkat and Ryser given in [4, Lemma 6.1, p. 353] (cf. [5, p. 498]) and also those of Minc given in [5, Theorems 3-5, pp. 501-502].

\section{REFERENCES}

1. K. M. Chong, Some extensions of a theorem of Hardy, Littlewood and Pólya and their applications, Canad. J. Math., XXVI (1974), 1321-1340.

2. K. M. Chong and N. M. Rice, Equimeasurable Rearrangements of Functions, Queen's Papers in Pure and Applied Mathematics, No. 28, 1971.

3. G. H. Hardy, J. E. Littlewood and G. Pólya, Some simple inequalities satisfied by convex functions, Mess. of Math., 58 (1929), 145-152.

4. W. B. Jurkat and H. J. Ryser, Term ranks and permanents of non-negative matrices, J. Algebra, 5 (1967), 342-357.

5. H. Minc, Rearrangements, Trans. Amer. Math. Soc., 159 (1971), 497-504.

Received November 18, 1974.

UNIVERSITY OF MALAYA 


\section{PACIFIC JOURNAL OF MATHEMATICS}

\section{EDITORS}

RICHARD ARENS (Managing Editor) University of California

Los Angeles, California 90024

\section{R. A. Beaumont}

University of Washington Seattle, Washington 98105
J. DugundJI Department of Mathematics University of Southern Californıa Los Angeles, California 90007

D. Gilbarg aNd J. Milgram Stanford University Stanford, California 94305

\section{ASSOCIATE EDITORS}

E. F. BeCKENBACH

B. H. NeUmanN

F. Wolf

K. YoSHIDA

\section{SUPPORTING INSTITUTIONS}

UNIVERSITY OF BRITISH COLUMBIA CALIFORNIA INSTITUTE OF TECHNOLOGY UNIVERSITY OF CALIFORNIA MONTANA STATE UNIVERSITY UNIVERSITY OF NEVADA NEW MEXICO STATE UNIVERSITY OREGON STATE UNIVERSITY UNIVERSITY OF OREGON OSAKA UNIVERSITY
UNIVERSITY OF SOUTHERN CALIFORNIA STANFORD UNIVERSITY UNIVERSITY OF TOKYO UNIVERSITY OF UTAH WASHINGTON STATE UNIVERSITY UNIVERSITY OF WASHINGTON AMERICAN MATHEMATICAL SOCIETY NAVAL WEAPONS CENTER 


\section{Pacific Journal of Mathematics \\ Vol. 59, No. 1 \\ May, 1975}

Shashi Prabha Arya and M. K. Singal, More sum theorems for topological

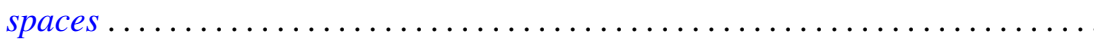

Goro Azumaya, F. Mbuntum and Kalathoor Varadarajan, On M-projective and

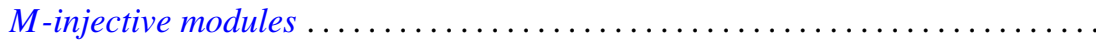

Kong Ming Chong, Spectral inequalities involving the infima and suprema of

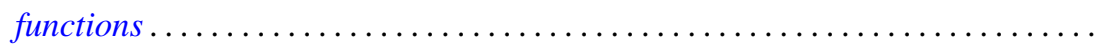

Alan Hetherington Durfee, The characteristic polynomial of the monodromy .......

Emilio Gagliardo and Clifford Alfons Kottman, Fixed points for orientation

preserving homeomorphisms of the plane which interchange two points ......

Raymond F. Gittings, Finite-to-one open maps of generalized metric spaces .......

Andrew M. W. Glass, W. Charles (Wilbur) Holland Jr. and Stephen H. McCleary,

$a^{*}$-closures of completely distributive lattice-ordered groups .............

Matthew Gould, Endomorphism and automorphism structure of direct squares of

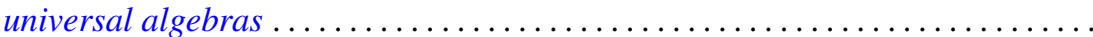

R. E. Harrell and Les Andrew Karlovitz, On tree structures in Banach spaces .....

Julien O. Hennefeld, Finding a maximal subalgebra on which the two Arens

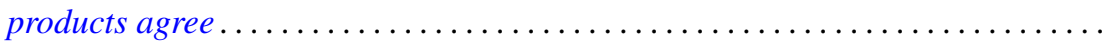

William Francis Keigher, Adjunctions and comonads in differential algebra .......

Robert Bernard Kelman, A Dirichlet-Jordan theorem for dual trigonometric

series

Allan Morton Krall, Stieltjes differential-boundary operators. III. Multivalued operators-linear relations...

Hui-Hsiung Kuo, On Gross differentiation on Banach spaces .

Tom Louton, A theorem on simultaneous observability ...

Kenneth Mandelberg, Amitsur cohomology for certain extensions of rings of algebraic integers.

Coy Lewis May, Automorphisms of compact Klein surfaces with boundary . . .

Peter A. McCoy, Generalized axisymmetric elliptic functions .

211

Muril Lynn Robertson, Concerning Siu's method for solving $y^{\prime}(t)=F(t$, $y(g(t)))$. .

Richard Lewis Roth, On restricting irreducible characters to normal

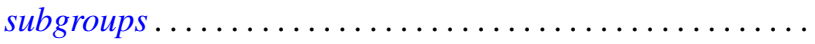

Albert Oscar Shar, $P$-primary decomposition of maps into an $H$-space .

Kenneth Barry Stolarsky, The sum of the distances to certain pointsets on the unit circle.

Bert Alan Taylor, Components of zero sets of analytic functions in $C^{2}$ in the unit ball or polydisc

Michel Valadier, Convex integrands on Souslin locally convex spaces ...

Januario Varela, Fields of automorphisms and derivations of $C$

Arnold Lewis Villone, A class of symmetric differential operators with deficiency

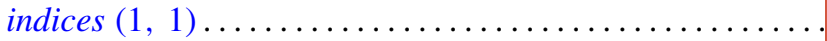

